\title{
Is there an equivalence of non-invasive to invasive referenciation in computer-aided surgery?
}

\author{
Tanja D. Grauvogel · Juergen Grauvogel • \\ Susan Arndt • Ansgar Berlis · Wolfgang Maier
}

\begin{abstract}
Various navigation systems with non-invasive patient referenciation and registration methods have been developed in times of minimal-invasive and computeraided surgery. However, hard data proving the equivalence of different referenciation systems are missing. The present study investigated invasive and non-invasive referenciation systems with regard to overall navigation accuracy as well as navigation accuracy at specific anatomic locations. Four skull models were individually fabricated with a 3D printer based on patient's CT data sets and fitted with an individual customized silicone skin. 26 titanium screws on defined anatomic locations served as target fiducials. Two noninvasive referenciation systems (headband and headset) were compared with the invasive skull fixed reference array. Registration was done with laser surface scan. The mean accuracy was calculated and the target registration error for eight anatomical locations was measured. Mean accuracy was $1.3 \pm 0.12 \mathrm{~mm}$ for the skull fixed reference array, $1.44 \pm 0.24 \mathrm{~mm}$ for the headset and $1.46 \pm$ $0.15 \mathrm{~mm}$ for the headband referenciation (non-significant). Navigation accuracy of the invasive referenciation system
\end{abstract}

T. D. Grauvogel $(\bowtie) \cdot S$. Arndt · W. Maier Department of Otorhinolaryngology-Head and Neck Surgery, Albert-Ludwigs-University Medical School, Killianstr. 5, 79106 Freiburg, Germany

e-mail: tanja.grauvogel@uniklinik-freiburg.de

J. Grauvogel

Department of Neurosurgery, Albert-Ludwigs-University

Medical School, Freiburg, Germany

A. Berlis

Department of Diagnostic Radiology and Neuroradiology, Medical School Augsburg, Augsburg, Germany was significantly superior to the accuracy of both noninvasive systems on the ethmoid sinus with respect to the selected anatomic locations. In the midface headband referenciation was statistically significantly worse than the invasive system. Invasive and non-invasive referenciation systems seem to be on par in terms of overall navigation accuracy, but not regarding specific anatomic locations. Therefore, invasive referenciation systems should be preferred in high precision surgery.

\section{Introduction}

There has been continuous further development since the simultaneous introduction of frameless stereotaxy in Germany, Japan, and the USA in 1986 [1]. Today Computeraided surgery (CAS) is a widespread tool in neuro-, craniomaxillo-facial-, orthopedic-, and ENT-Surgery. Various different detection systems based on optical, electromechanical, electromagnetic, or ultrasound measurements are available.

Since operative techniques have changed over the last years from wide-open to minimal-invasive procedures, CAS has gained importance and numerous non-invasive navigation tools have been developed to follow the trend.

Beside different non-invasive registration modalities (skin-fixed fiducial markers, anatomic landmarks, oral splint, laser scan) non-invasive referenciation trackers (headset, headband, oral splint) are available which are of special interest for ENT-surgery because they allow unimpeded head movement. 
In clinical application, navigation systems reach navigation accuracies with target registration errors (TRE) between 0.5 and $2.77 \mathrm{~mm} \mathrm{[2-4]} \mathrm{and} \mathrm{are} \mathrm{considered} \mathrm{by}$ most surgeons to be a helpful tool [5]. However, most studies examine only one referenciation or registration modality [6-9]. Other publications only focus on overall navigation accuracy, whereas differences in accuracy at specific anatomic locations are often not taken into consideration $[9,10]$. But especially in high precision surgery of the skull base a difference in navigation accuracy depending on the anatomic location is of special interest for the surgeon due to the complex anatomy. As navigation accuracy is influenced by many factors we examined for the first time whether the use of a non-invasive referenciation system reduces navigation accuracy compared to an invasive-system. Individually fabricated skull models were used for the measurement of overall navigation accuracy as well as accuracy measurements at specific anatomic locations. Skull model registration was performed with laser scan.

\section{Materials and methods}

Four skull models consisting of a special mixture of powder, binder, and infiltrant (ZP130/ZB7/Z-Bond 101, Z Corporation, Burlington, USA) and covered with an individual silicone skin were used for accuracy measurements. Skull model fabrication was done with the help of a 3D printer (Spectrum $\mathrm{Z}^{\mathrm{TM}} 510, \mathrm{Z}$ Corporation, Burlington, USA) based on CT scans of real patients. The specially customized silicone skin with an individual surface as used by make-up artists in the theatre (Fig. 1) was fictitious and independent of facial contours of real patients.

26 titanium screws (length $5 / 17 \mathrm{~mm}$, diameter $1 \mathrm{~mm}$, square cavity $1 \mathrm{~mm}$, Stryker-Leibinger, Freiburg, Germany) bilaterally distributed over each skull served as target fiducials. To guarantee precise marker positioning and pointer actuation in the anterior and lateral skull base, a part of the cranium was left open during fabrication (Fig. 1).

All skulls underwent CT scan (Somatom Sensation 16, Siemens AG, Munich, Germany) with a slice thickness of $1 \mathrm{~mm}$, gantry $0^{\circ}$, resolution $(\mathrm{X} \times \mathrm{Y}) 512 \times 512$, and pixel size $(\mathrm{mm}) 0.396 \times 0.396$. After import of the DICOM data sets into the navigation software, all titanium screws were marked in the coronal, axial, and sagittal slices with a special software program (iPlan cranial 2.5, BrainLAB AG, Feldkirchen, Germany). With the help of the Vector Vision ${ }^{\circledR}$ (BrainLAB AG, Feldkirchen, Germany) navigation system, three different referenciation modalities were examined in the study: (1) the invasive skull fixed reference array (SRA), (2) the non-invasive headset (HS), (3) the non-invasive headband (HB). According to the experimental set-up, either the SRA was screwed into the skull close behind the frontal hairline (comparable to clinical conditions) or the HB or the HS was fixed on the skull model (Fig. 1).

The registration procedure with laser surface scanning was repeated five times per skull. After each registration, each target fiducial was actuated five times with the navigation pointer (Fig. 2). The squared cavity in each screw allowed exact marker positioning. The virtual and the real coordinates of each fiducial $(x, y, z$-coordinates) were displayed by the navigation system. The Euclidian distance could then be calculated for each target fiducial which is consistent with the TRE $d(x, y, z)=\sqrt{\Delta x^{2}+\Delta y^{2}+\Delta z^{2}}$.

The experiments were carried out by two independent operators and took place in the operating room comparable to clinical conditions.

Data are expressed as quadratic mean values $(\mathrm{mm}) \pm$ standard deviation $(\mathrm{mm})$. Statistical analysis was performed with Tukey Test and variance analysis. A probability $p<0.05$ was considered statistically significant.
Fig. 1 Individually fabricated skull models based on patients' CT data sets and coated with a silicone skin mounted with skull fixed reference array (left), headset (middle), and headband (right) as different referenciation systems. Titanium screws drilled in the skull and skull base serve as target fiducials
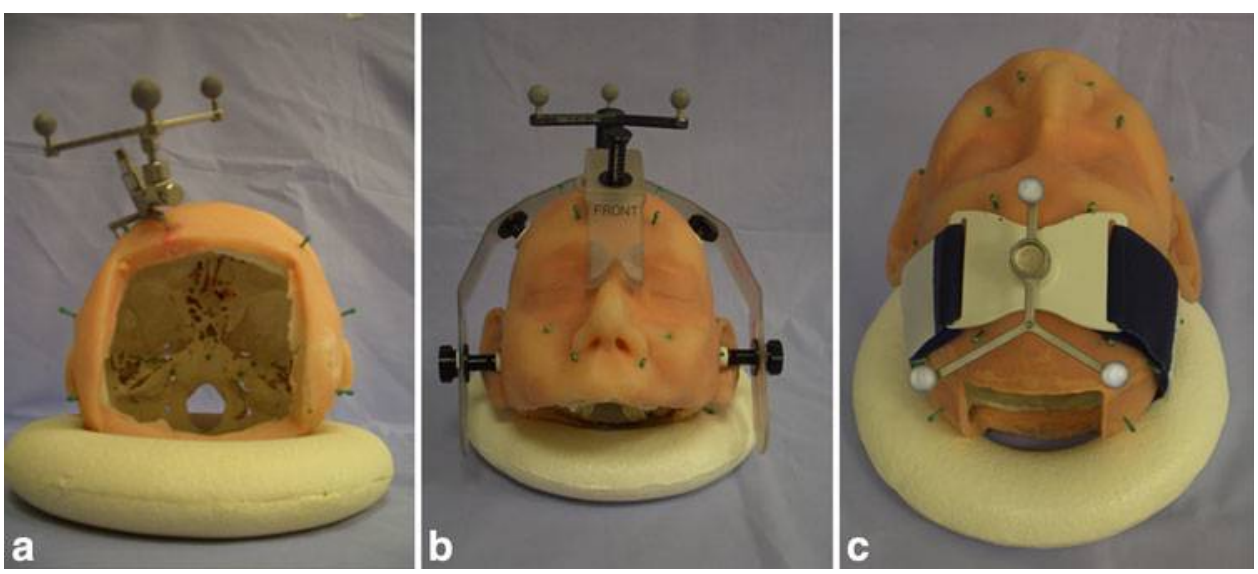


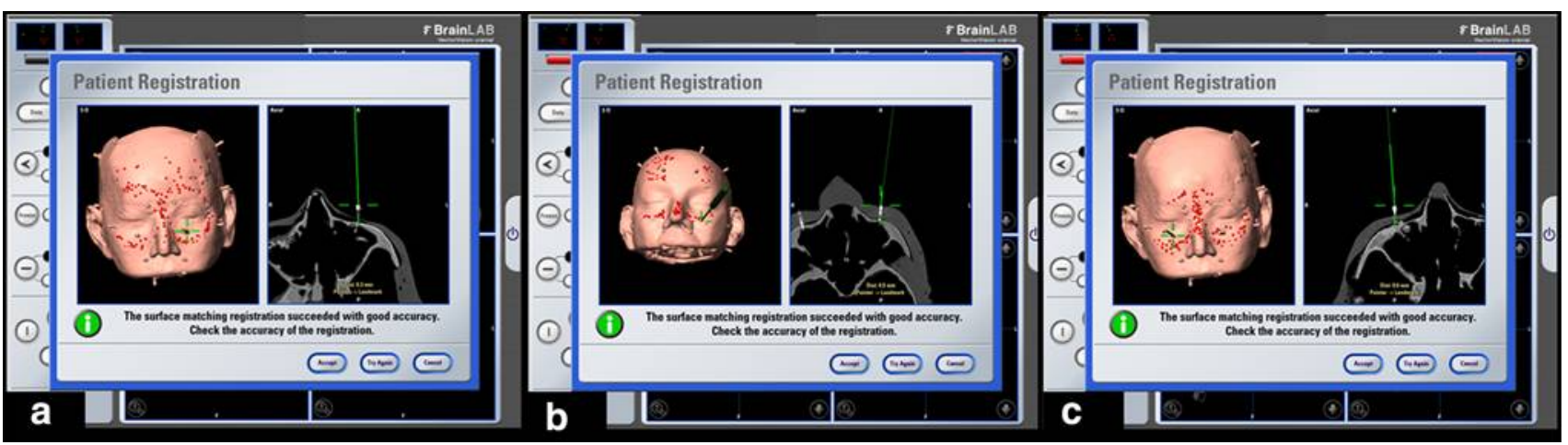

Fig. 2 Screenshots after laser surface scanning; referenciation with the skull fixed reference array (left), with the headset (middle), and with the headband (right); accuracy measurements by actuating the titanium screws with the navigation pointer

The study was approved by the Institutional Review Board (Research Commission of the Faculty of Medicine) of the Albert-Ludwigs-University at Freiburg, Germany.

\section{Results}

The extent of the detected surface after laser scanning varied in dependence on the different referenciation systems (Fig. 2). As Fig. 2 demonstrates the HS had a hidden zone at the nasion and the centre of the frontal bone. The undetected zone of the HB was bilateral in the frontal and temporal bone (Fig. 2).

The mean navigation accuracy (with respect to all 26 titanium screws) after laser surface registration was $1.30 \pm 0.12 \mathrm{~mm}$ (quadratic mean $\pm \mathrm{SD}$ ) with the SRA, $1.44 \pm 0.24 \mathrm{~mm}$ with the HS, and $1.46 \pm 0.15 \mathrm{~mm}$ with the HB for skull model referenciation (Table 1). There were no significant differences in mean TRE between the three groups.

TREs at selected locations of special interest for the ENT surgeon are given in Fig. 3. Mean values for SRA referenciation showed the lowest TRE in nearly all locations compared to HS and HB referenciation. However, statistically significant differences in navigation accuracy could only be found in the midface and frontal skull base.

Table 1 Overall navigation accuracy for three different referenciation systems

\begin{tabular}{lllll}
\hline $\begin{array}{l}\text { Referenciation } \\
\text { system }\end{array}$ & $\begin{array}{l}\text { Minimum } \\
\text { TRE }(\mathrm{mm})\end{array}$ & $\begin{array}{l}\text { Maximum } \\
\text { TRE }(\mathrm{mm})\end{array}$ & $\begin{array}{l}\text { Mean } \\
\text { TRE }(\mathrm{mm})\end{array}$ & $\begin{array}{l}\text { SD } \\
(\mathrm{mm})\end{array}$ \\
\hline SRA & 1.00 & 1.50 & 1.30 & 0.12 \\
HS & 0.91 & 1.93 & 1.44 & 0.24 \\
HB & 1.17 & 1.68 & 1.46 & 0.15 \\
\hline
\end{tabular}

TRE target registration error: quadratic mean \pm standard deviation (SD) in mm, $S R A$ skull fixed reference array, $H S$ headset, $H B$ headband; Tukey test and variance analysis

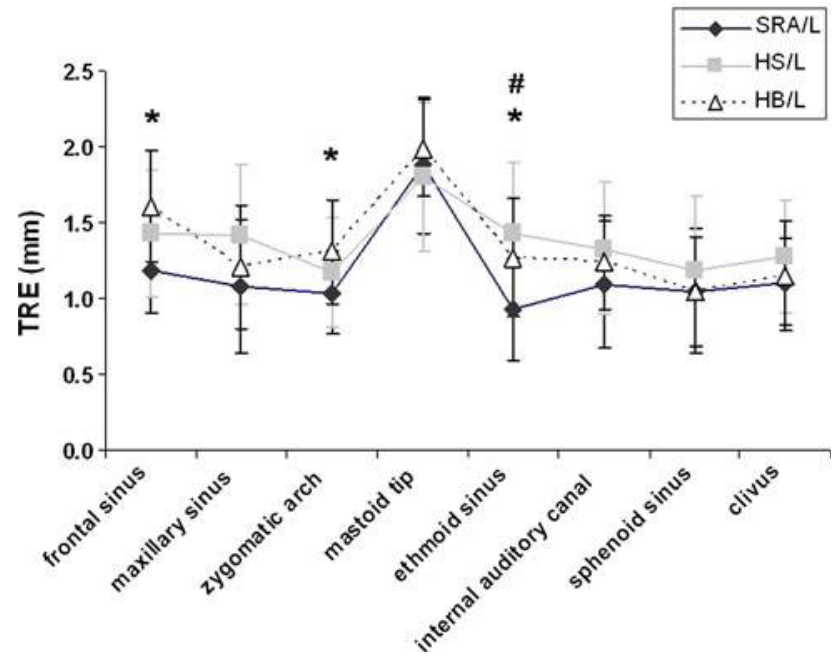

Fig. 3 Target registration error (TRE) at eight selected locations: quadratic mean \pm standard deviation in $\mathrm{mm}$ after laser surface scanning with three different referenciation systems (skull fixed reference array/laser (SRA/L), headset/laser (HS/L), headband/laser (HB/L); *SRA/L vs. HB/L; " $\mathrm{SRA} / \mathrm{L}$ vs. HS/L; $p<0.05$; Tukey test and variance analysis

In detail, SRA referenciation was found to be significantly superior to HS and HB referenciation with regard to the ethmoid sinus. In addition, referenciation with the HB was statistically significantly worse in the central and lateral midface (frontal sinus and zygomatic arch) compared to referenciation with the SRA.

The comparison of the two non-inavsive referenciation methods (HB and HS) showed no significant differences at selected anatomic locations.

\section{Discussion}

The era of minimal-invasive surgery issues a great challenge to surgeons. Beside the claim of complete tumor resection or successful and symmetric reconstruction, the surgeon should avoid big scars, reach best possible 
aesthetic outcomes, and shorten hospitalization time. CAS as a supportive tool follows this trend and is becoming less and less invasive.

But the use of a navigation system has to meet certain demands in order to be an aid to the surgeon and increase efficacy and safety. Particularly in the field of otolaryngology there are areas of surgical intent that frequently exhibit close proximity to critical anatomical structures. Therefore, a high-system accuracy in the $1.0-$ to $2.0-\mathrm{mm}$ range is mandatory for rhinological procedures [11]. In lateral skull base surgery, a navigation accuracy $<1 \mathrm{~mm}$ would be desirable as the petrous bone presents complexity in three-dimensional arrangement.

A frameless tracking device which allows unimpeded head movement would be favorable especially in ENT surgery. Clinical studies have shown that available navigation systems meet most of these requirements, but there is considerable variation in navigation accuracy depending on different anatomic regions and different registration modalities $[3,4,12]$.

Overall navigation accuracy is influenced by many factors $[13,14]$. Although data acquisition usually follows predetermined protocols, there might be a loss in navigation accuracy due to incomplete CT or MRT data sets, dental or movement artifacts, and soft tissue deformity.

Each of the various detection modalities of navigation systems available is associated with certain terms and conditions guaranteeing the highest possible navigation accuracy. For example, ferromagnetic instruments in the magnetic field of electromagnetic navigation systems cause field distortion and a lack of accuracy $[1,15]$. The use of optical-based systems requires a certain distance from the patient tracker to the camera as well as attention to the line of sight for best navigation results $[1,16]$.

The great number of influencing factors on navigation accuracy cannot be examined within one study. For this reason, in the present study, we exclusively examined whether or not there are differences in navigation accuracy on specific anatomic locations due to the chosen reference tool. All other workflows like data acquisition, registration, and accuracy measurement were standardized in all groups to avoid other sources of error. Here, we concentrated on overall navigation accuracy as well as on navigation accuracy on specific anatomic landmarks. Titanium screws were used as target fiducials since they guarantee best possible accuracy measurements.

Most studies examine the influence of a single or different registration methods on navigation accuracy [6-9, 17]. Clinical or laboratory examinations comparing different referenciation systems do not exist. Other publications mainly focus on overall navigation accuracy, and differences in accuracy at specific anatomic locations are often not taken into consideration.
Today, a variety of referenciation systems is available. A tracker fixed to the Mayfield clamp has become established in Neurosurgery. Over the past years, Cranio-Maxillo-Facial and ENT surgeons have looked for patient trackers which allow unimpeded head movement. Beside skull fixed reference arrays, the use of non-invasive systems is increasing. Currently available non-invasive systems are headsets, headbands, oral splints, and LED masks.

The aim of the present study was to examine the influence of different referenciation methods on navigation accuracy. Our study is the first study that compares the overall accuracy as well as the accuracy in different surgical fields using an SRA, an HS, or an HB for referenciation. Registration was performed with laser surface scanning in all cases.

We used individually fabricated skull models based on CT scans of real patients to avoid the use of cadavers. Unlike industrially manufactured skull models, which are often used in navigation studies $[6,18]$, our custom-made skulls differed from each other in size and anatomy comparable to human beings. Although skull fabrication requires time and money, the models are durable and very versatile. The individually fitted silicone mask for skin permitted a realistic facial outline with nose and cheek, which is of special interest for laser surface registration. With this setup, we achieved a registration situation which is very close to the real procedure and the intraoperative circumstances during patient registration. However, the influence on navigation accuracy of skin surface alterations caused by edema, tumor, or skin turgor could not be imitated by this method.

These influencing factors can only be avoided by using invasive fiducial marker registration, which is still the gold standard reaching highest navigation accuracy [17, 19]. However, since surgical interventions become more and more less invasive, this registration method is no longer used in daily routine, particularly when non-invasive reference tools are applied.

Additional influencing factors such as washing and preparation of the surgical field, sterile covering of the patient, intraoperative movement of the head or even unintentional touching of the reference matrix were not examined in our study. These factors, which can hardly be standardized, are obvious influencing factors resulting in heavy losses of navigation accuracy or even rendering navigation completely unusable. Because the primary goal of the present study was to compare maximal achievable navigation accuracy of three different referenciation tools for different anatomic locations these factors have not been examined in this study.

We chose laser surface scanning for phantom registration because it is a non-invasive registration method that uses the anatomy of the patient's head and face by scanning 
it with a laser beam. Furthermore, this is a frequently used strategy in clinical settings. The need for second planning imaging can be avoided using this registration technique.

The currently available systems are the Fazer, which acquires 300 points during a surface scan (StealthStation Treon; Medtronic, Inc.) and the z-touch (200 points), which was used in our study. Surface scanning of the midface is recommended for best accuracy results. Grevers et al. [20] considered patient registration with the z-touch as a fast and simple method with a navigation accuracy $<2.5 \mathrm{~mm}$. Other study groups reported similar results in the clinical setting [3, 4]. The mean accuracy value of $1.3 \pm 0.12 \mathrm{~mm}$ obtained by laser surface registration with the SRA in our study is comparable to accuracies measured in previous experimental studies using the same navigation system and the SRA for patient tracking $[8,21,22]$. Direct comparison of these experimental results with clinical studies is not possible. Anatomic landmarks are used for accuracy measurements under clinical conditions. These anatomic target fiducials cannot be as precise as on the basis of screws, because the vast majority of landmarks are not exactly defined. Laser surface scanning with the Fazer reached accuracies of about $1.8 \mathrm{~mm}$ in cadaver studies [10].

Marmulla et al. [23] tested alternative anatomic structures for laser surface scanning. Clinical studies using the upper jaw for laser scanning showed TRE values of $0.8 \pm 0.3 \mathrm{~mm}$, whereas the lower jaw proved to be inapplicable for this registration method. To improve navigation accuracy in the lateral skull base, they also did laser scan of the auricle. With this method, a mean TRE of $0.9 \pm 0.3 \mathrm{~mm}$ periauricular was attained as long as the auricle was not deformed during $\mathrm{CT}$ imaging or laser scanning. There are only few studies examining navigation accuracy at specific anatomic locations.

Raabe et al. [4] reported values between 5.0 and $1.9 \mathrm{~mm}$ on the nasion and between 10.3 and 0.8 on the external auditory canal. Schlaier et al. [3] reported TRE of $2.61 \pm 2.06$ (distance to tragus), $2.19 \pm 1.98$ (distance to canthus lat.) and $2.77 \pm 1.64$ to any target fiducial. Overall there is a great range of the measured accuracy values, because most studies use anatomic landmarks as target fiducials which cannot be actuated as precisely as screws or other fixed markers with the navigation pointer.

Our measurements at different anatomical locations using laser surface scanning in combination with the SRA are mainly consistent with those measured by Ledderose and coworkers [8] on cadavers. The better results at the internal auditory canal ( $1.09 \mathrm{~mm}$ vs. 1.76 petrous bone) are probably due to better actuation of the titanium screw through the opening of the calvarium in our study. Troitsch et al. [22] used one titanium screw lateroparietal to obtain a target difference value of $2.08+0.49$ with the $z$-touch. However, in that study, as well as in the study by Luebbers et al. [21], there was no skin simulation.

Literature about the application of headsets and their accuracy is sparse. Most authors dealing with headset referenciation use the electromagnetic system InstaTrak, which additionally offers the possibility of automatic registration. For example Javer et al. and Ecke et al. [24, 25] measured accuracy values between 0.69 and $1.89 \mathrm{~mm}$ with this navigation system. However, both studies used automatic registration so that their results cannot be directly compared with the TREs measured in our study using the laser scan for patient registration.

Knott and coworkers [18] used the InstaTrak headset in combination with a contour-based registration protocol with 500, 250, 125, 50, and 4 points. Mean TRE values ranged between 1.5 and $1.8 \mathrm{~mm}$ at the anterior ethmoid, and 2.6 and $1.5 \mathrm{~mm}$ at the sphenoid face depending on the registration protocol. Interestingly, contour-based registration with as few as 50 points revealed best TRE values of $1.5 \mathrm{~mm}$, which are comparable to our results at corresponding locations.

The headset used in our study is no longer fabricated by BrainLAB and can be considered as precursor of the laterdeveloped headband. In our clinic, we have routinely used the headset for several years during navigated operations with good experience. We also included it in our experimental study to see whether the HB is equivalent to its precursor, the HS. Our results show that there is an equivalence of five anatomic locations compared to the SRA, but that the HB is significantly worse in three, the HS only in one location.

There are also only few literature data available regarding patient referenciation with the headband. Balachandran and co-workers [26] reported TRE values in the region of the lateral skull base of $3.21 \pm 1.02 \mathrm{~mm}$ after laser surface scanning. They used a customized post attached to a BAHA abutment for their measurements. A steel ball was affixed onto this post to serve as target fiducial. In our study, we found an accuracy of $1.99 \pm 0.32 \mathrm{~mm}$ at the mastoid and $1.24 \pm 0.31 \mathrm{~mm}$ at the internal auditory canal when using the headband. Our better results might be explained by better identification of the titanium screw compared to the steel ball on CT dataset as well as during pointer actuation.

Headsets and headbands for patient referenciation have the advantage of being non invasive. In our clinical experience, installation time of these referenciation tools is shorter than that of the invasive system. But due to their non-invasive fixation, they might dislocate more easily and their configuration determines the reference star position. In addition, the headband and the headset offer less skin surface for the registration process which might contribute to the loss of accuracy in case of laser surface scanning, 
especially at the anterior skull base for both systems and in case of the headset also more lateral at the zygomatic arch as our measurement results show (Figs. 2, 3).

\section{Conclusion}

With an overall navigation accuracy of $<1.5 \mathrm{~mm}$, noninvasive referenciation systems seem to be equivalent alternatives to their invasive counterparts. But differences in location-specific accuracy require a differentiated approach. We would recommend the use of an invasive referenciation system in high precision surgery, especially in the anterior and lateral skull base.

Acknowledgments The authors thank Professor Dr. J. SchulteMoenting from the Institute of Biostatistics and Medical Informatics, University of Freiburg, Germany for his support in statistical analysis and Ms I. Neu, make-up artist at the theatre of Freiburg, Germany for fabricating the silicone masks. This work was supported by the Faculty of Medicine, Albert-Ludwigs-University of Freiburg, Germany.

Conflict of interest The authors declare that they have no conflict of interest.

\section{References}

1. Wise SK, DelGaudio JM (2005) Computer-aided surgery of the paranasal sinuses and skull base. Expert Rev Med Devices 2(4):395-408

2. Caversaccio M, Nolte LP, Hausler R (2002) Present state and future perspectives of computer aided surgery in the field of ENT and skull base. Acta Otorhinolaryngol Belg 56(1):51-59

3. Schlaier J, Warnat J, Brawanski A (2002) Registration accuracy and practicability of laser-directed surface matching. Comput Aided Surg 7(5):284-290

4. Raabe A, Krishnan R, Wolff R, Hermann E, Zimmermann M, Seifert V (2002) Laser surface scanning for patient registration in intracranial image-guided surgery. Neurosurgery 50(4):797-801 (discussion 802-793)

5. Citardi MJ, Batra PS (2007) Intraoperative surgical navigation for endoscopic sinus surgery: rationale and indications. Curr Opin Otolaryngol Head Neck Surg 15(1):23-27

6. Eggers G, Muhling J (2007) Template-based registration for image-guided skull base surgery. Otolaryngol Head Neck Surg 136(6):907-913

7. Labadie RF, Shah RJ, Harris SS, Cetinkaya E, Haynes DS, Fenlon MR, Juszczyk AS, Galloway RL, Fitzpatrick JM (2005) In vitro assessment of image-guided otologic surgery: submillimeter accuracy within the region of the temporal bone. Otolaryngol Head Neck Surg 132(3):435-442

8. Ledderose GJ, Stelter K, Leunig A, Hagedorn H (2007) Surface laser registration in ENT-surgery: accuracy in the paranasal sinuses-a cadaveric study. Rhinology 45(4):281-285

9. Metzger MC, Rafii A, Holhweg-Majert B, Pham AM, Strong B (2007) Comparison of 4 registration strategies for computer-aided maxillofacial surgery. Otolaryngol Head Neck Surg 137(1):93-99
10. Schicho K, Figl M, Seemann R, Donat M, Pretterklieber ML, Birkfellner W, Reichwein A, Wanschitz F, Kainberger F, Bergmann H, Wagner A, Ewers R (2007) Comparison of laser surface scanning and fiducial marker-based registration in frameless stereotaxy. Technical note. J Neurosurg 106(4):704-709

11. Cartellieri M, Vorbeck F (2000) Endoscopic sinus surgery using intraoperative computed tomography imaging for updating a three-dimensional navigation system. Laryngoscope $110(2 \mathrm{Pt}$ 1):292-296

12. Greenfield JP, Howard BM, Huang C, Boockvar JA (2008) Endoscopic endonasal transsphenoidal surgery using a skull reference array and laser surface scanning. Minim Invasive Neurosurg 51(4):244-246

13. Ecke U, Maurer J, Boor S, Khan M, Mann WJ (2003) Common errors of intraoperative navigation in lateral skull base surgery. HNO 51(5):386-393

14. Widmann G, Stoffner R, Bale R (2009) Errors and error management in image-guided craniomaxillofacial surgery. Oral Surg Oral Med Oral Pathol Oral Radiol Endod 107(5):701-715

15. Gunkel AR, Thumfart WF, Freysinger W (2000) Computer-aided 3D-navigation systems. Survey and location determination. HNO 48(2):75-90

16. Caversaccio, Freysinger (2003) Computer assistance for intraoperative navigation in ENT surgery. Minim Invasive Ther Allied Technol 12(1):36-51

17. Grauvogel TD, Soteriou E, Metzger MC, Berlis A, Maier W (2010) Influence of different registration modalities on navigation accuracy in ear, nose, and throat surgery depending on the surgical field. Laryngoscope 120(5):881-888

18. Knott PD, Batra PS, Butler RS, Citardi MJ (2006) Contour and paired-point registration in a model for image-guided surgery. Laryngoscope 116(10):1877-1881

19. Mascott CR, Sol JC, Bousquet P, Lagarrigue J, Lazorthes Y, Lauwers-Cances V (2006) Quantification of true in vivo (application) accuracy in cranial image-guided surgery: influence of mode of patient registration. Neurosurgery 59(1 Suppl 1):ONS146-156 (discussion ONS146-156)

20. Grevers G, Leunig A, Klemens A, Hagedorn H (2002) CAS of the paranasal sinuses-technology and clinical experience with the Vector-Vision-Compact-System in 102 patients. Laryngorhinootologie 81(7):476-483

21. Luebbers HT, Messmer P, Obwegeser JA, Zwahlen RA, Kikinis R, Graetz KW, Matthews F (2008) Comparison of different registration methods for surgical navigation in cranio-maxillofacial surgery. J Craniomaxillofac Surg 36(2):109-116

22. Troitzsch D, Hoffmann J, Dammann F, Bartz D, Reinert S (2003) Registration using three-dimensional laser surface scanning for navigation in oral and craniomaxillofacial surgery. Zentralbl Chir 128(7):551-556

23. Marmulla R, Eggers G, Muhling J (2005) Laser surface registration for lateral skull base surgery. Minim Invasive Neurosurg 48(3):181-185

24. Javer AR, Kuhn FA, Smith D (2000) Stereotactic computerassisted navigational sinus surgery: accuracy of an electromagnetic tracking system with the tissue debrider and when utilizing different headsets for the same patient. Am J Rhinol 14(6):361-365

25. Ecke U, Luebben B, Maurer J, Boor S, Mann WJ (2003) Comparison of different computer-aided surgery systems in skull base surgery. Skull Base 13(1):43-50

26. Balachandran R, Fitzpatrick JM, Labadie RF (2008) Accuracy of image-guided surgical systems at the lateral skull base as clinically assessed using bone-anchored hearing aid posts as surgical targets. Otol Neurotol 29(8):1050-1055 